\title{
Improved neutralization of the SARS-CoV-2 Omicron variant after Pfizer-BioNTech BNT162b2 COVID-19 vaccine boosting
}

Kerri Basile $\dagger^{1}$, Rebecca J Rockett ${ }^{2,4}$, Kenneth McPhie $^{1,3}$, Michael Fennell ${ }^{1}$, Jessica JohnsonMackinnon $^{2}$, Jessica E Agius ${ }^{2}$, Winkie Fong ${ }^{2}$, Hossinur Rahman ${ }^{1}$, Danny Ko ${ }^{1}$, Linda Donavan ${ }^{1}$, Linda Hueston ${ }^{1}$, Connie Lam ${ }^{2}$, Alicia Arnott ${ }^{1}$, Sharon C-A Chen ${ }^{1,2,4}$, Susan Maddocks ${ }^{1}$, Matthew V O’Sullivan ${ }^{1,2,4}$, Dominic E Dwyer ${ }^{1,2,4}$, Vitali Sintchenko ${ }^{1,2,4}$, and Jen Kok*1,2,4

1. Centre for Infectious Diseases and Microbiology Laboratory Services, NSW Health Pathology Institute of Clinical Pathology and Medical Research, Westmead Hospital, Westmead New South Wales 2145, Australia

2. Centre for Infectious Diseases and Microbiology - Public Health, Westmead Hospital, Westmead New South Wales 2145, Australia

3. Westmead Institute for Medical Research, Westmead, New South Wales 2145, Australia

4. Sydney Institute for Infectious Diseases, The University of Sydney New South Wales 2006,

Australia

† Joint first authors

*Corresponding author:

Dr Jen Kok jen.kok@health.nsw.gov.au

Centre for Infectious Diseases and Microbiology Laboratory Services

Westmead Hospital Westmead New South Wales 2145 AUSTRALIA

\section{Abstract}

In late November 2021, the World Health Organization declared the SARS-CoV-2 lineage B.1.1.529 the fifth variant of concern, Omicron. This variant has acquired 15 mutations in the receptor binding domain of the spike protein, raising concerns that Omicron could evade naturally acquired and vaccinederived immunity. We utilized an authentic virus, multicycle neutralisation assay to demonstrate that sera collected 1, 3, and 6 months post-two doses of Pfizer-BioNTech BNT162b2 has a limited ability to neutralise SARS-CoV-2. However, four weeks after a third dose, neutralizing antibody titres are boosted. Despite this increase, neutralising antibody titres are reduced 4-fold for Omicron compared to lineage A.2.2 SARS-CoV-2.

\section{Introduction}

In November 2021, the SARS-CoV-2 Omicron (B.1.1.529) variant of concern (VOC) emerged in Gauteng province, South Africa, coinciding with a rapid rise in COVID-19 cases. The World Health Organization designated Omicron a VOC two days following its identification. ${ }^{1,2}$ There has been subsequent spread worldwide with Omicron cases now reported in over 50 countries. Early 
epidemiological reports from South Africa suggest that Omicron has an increased ability to evade prior infection-induced immunity compared with the Delta and Beta VOC. ${ }^{1}$ Questions regarding Omicron's ability to evade vaccine-derived immunity were also raised following transmission between two vaccinated individuals whilst in hotel quarantine. ${ }^{2}$ Omicron is characterized by over 30 non-synonymous mutations in the spike protein (e.g. E484A, K417N, P681H, N501Y, T478K), many of which are within key epitopes that provide SARS-CoV-2 an advantage over host immune responses, but these are yet to be fully quantified in vitro. Here we present data outlining the reduced ability of post-two and three dose vaccination sera to neutralize Omicron compared to Delta and wildtype lineages, but with improvement after a third vaccine dose.

\section{Methods}

SARS-CoV-2 culture. Upper respiratory tract specimens collected in universal transport media (UTM) where SARS-CoV-2 RNA was detected by reverse transcriptase real-time polymerase chain reaction (RT-PCR) were used to inoculate VeroE6 expressing transmembrane serine protease 2 (TMPRSS2) [VeroE6/TMPRSS2; JCRB1819] cells as previously outlined. ${ }^{3}$

In brief, cells were seeded at $1-3 \times 10^{4}$ cells $/ \mathrm{cm}^{2}$ whilst in the log phase of replication with Dulbecco's minimal essential medium (DMEM) (Lonza) supplemented with 9\% foetal bovine serum (FBS) (HyClone Cytiva) and Geniticin ( $1 \mathrm{mg} / \mathrm{mL}$, Gibco) in $25 \mathrm{~cm}^{2}$ cell culture flasks (Corning Inc). The media was changed within $12 \mathrm{hrs}$ for inoculation media containing 1\% FBS and 1\% antimicrobials (amphotericin B deoxycholate $25 \mu \mathrm{g} / \mathrm{mL}$, penicillin 10,000 U/mL and streptomycin 10,000 $\mu \mathrm{g} / \mathrm{mL}$ ) (Lonza) to prevent microbial overgrowth and then inoculated with $500 \mu \mathrm{L}$ of clinical specimen into $25 \mathrm{~cm}^{2}$ cell culture flasks. Routine mycoplasma testing was performed to exclude mycoplasma contamination of the cell lines and all manipulation of SARS-CoV-2 cultures were performed under biosafety level 3 (BSL3) conditions.

Cultures were inspected daily for cytopathic effect (CPE); the inoculum and supernatant were sampled at $96 \mathrm{hrs}$ for SARS-CoV-2 in-house quantitative reverse transcriptase real time polymerase chain reaction (RT-qPCR) targeting the $N$-gene as previously described. ${ }^{4}$ A decrease in the cycle threshold (Ct) from the inoculum RT-qPCR result as well as the presence of CPE was used to determine the propagation of SARS-CoV-2. Viral culture supernatant was harvested $96 \mathrm{hrs}$ post-infection and stored at $-80^{\circ} \mathrm{C}$ in $500 \mu \mathrm{L}$ aliquots in $2 \mathrm{ml}$ cryovials (Sarstedt) until required. SARS-CoV-2 complete genomes were sequenced from the initial clinical specimen, positive culture supernatant, and post-neutralization (72 hrs) to quantify genomic variations that may have developed during propagation (Supplementary Table 2). 
SARS-CoV-2 viral load quantitation by qRT-PCR. A previously described RT-PCR 5 , targeting the nucleocapsid gene was employed to estimate the viral load of the viral inoculum and post-neutralisation viral culture. Serial (10-fold) dilutions starting at 20,000 copies $/ \mu \mathrm{L}$ to 2 copies $/ \mu \mathrm{L}$ of a commercially available synthetic RNA control (Wuhan-1 strain, TWIST Biosciences NCBI GenBank accession MN908947.3) were used to generate a standard curve and quantify the viral load of each culture extract. The mean cycle threshold of biological replicates was used to calculate the viral load. A positive change in the viral load between the viral inoculum ( 0 hours) and 72 hours post-neutralisation was used to indicate positive viral replication.

SARS-CoV-2 sequencing. Tiling PCR was used to amplify the entire SARS-CoV-2 genome from RNA extracts of clinical specimens using primers outlined in the Midnight sequencing protocol, the viral respiratory oligo panel (RVOP, Illumina) (Omicron), Artic v3 primers (Delta), or a previously described long amplicon methodology (Wild-type). ${ }^{5,6}$ Each PCR included 12.5 $\mu \mathrm{L}$ Q5 High Fidelity 2x Master Mix (New England Biolabs), 1.1 $\mu \mathrm{L}$ of either pool 1 or pool $210 \mu \mathrm{M}$ primer master mix, $2.5 \mu \mathrm{L}$ of template RNA and molecular grade water was added to generate a total volume of $25 \mu \mathrm{L}$. Cycling conditions were initial denaturation at $95^{\circ} \mathrm{C}$ for $2 \mathrm{~min}$, then 35 cycles of: $95^{\circ} \mathrm{C}$ for $30 \mathrm{~s}, 65^{\circ} \mathrm{C}$ for $2 \min 45 \mathrm{~s}$, and a final extension step of $75^{\circ} \mathrm{C}$ for $10 \mathrm{~min}$. Pool 1 and pool 2 amplicons were combined and purified with a 1:1 ratio of AMPureXP beads (Beckman Coulter) and eluted in $30 \mu \mathrm{L}$ of RNAase free water. Purified products were quantified using Qubit ${ }^{\mathrm{TM}}$ 1x dsDNA HS Assay Kit (Thermo Fisher Scientific) and diluted to the desired input concentration for library preparation. Sequencing libraries were prepared using Nextera XT (Illumina) according to the manufacturer's respective instructions and pooled with the aim of producing $1 \times 10^{6}$ reads per library. Sequencing libraries were then sequenced with paired end $76 \mathrm{bp}$ chemistry on the iSeq or MiniSeq (Illumina) platforms.

Bioinformatic analysis. Raw sequence data were processed using an in-house quality control procedure prior to further analysis as described previously. ${ }^{5,7}$ De-multiplexed reads were quality trimmed using Trimmomatic v0.36 (sliding window of 4, minimum read quality score of 20, leading/trailing quality of 5 and minimum length of 36 after trimming). ${ }^{8}$ Briefly, reads were mapped to the reference SARS-CoV-2 genome (NCBI GenBank accession MN908947.3) using Burrows-Wheeler Aligner (BWA)-mem version $0.7 .17^{9}$, with unmapped reads discarded. Average genome coverage was estimated by determining the number of missing bases (Ns) in each sequenced genome. Variant calling and the generation of consensus sequences was conducted using $\mathrm{iVar}^{10}$, with soft clipping over primer regions (version 1.2.1, 
min. read depth $>10 x$, quality $>20$, min frequency threshold of 0.1 ). Polymorphic sites that have previously been highlighted as problematic were monitored. ${ }^{11}$ SARS-CoV-2 lineages were inferred using Phylogenetic Assignment of Named Global Outbreak LINeages v1.2.86 (PANGO and PLEARN). ${ }^{12,13}$

Post-BNT162b2 vaccine sera. Sera were sourced from Australian healthcare workers caring for, or handling specimens from, individuals exposed to, or diagnosed with, SARS-CoV-2 infection enrolled in the COVID Heroes Serosurvey (http://www.covidheroes.org.au). Sera were tested upon receipt with an in-house immunofluorescence assay (IFA) against SARS-CoV-2-specific IgA, IgM and IgG and then stored at $4{ }^{\circ} \mathrm{C} .{ }^{14}$ Fourteen sera samples were included from an age and sex-matched cohort of 12 participants (Supplementary Table 1). This included a vaccine-naïve individual $(\mathrm{n}=1)$, individuals who received two doses of BNT162b2 $(\mathrm{n}=9)$ and individuals that received 6 months after the primary schedule $(n=4)$. Median age was 59 years [Range 34-65] with sera collected 1 month, 3 months, 6 months post-primary vaccination and 4 weeks after the third dose. All vaccine recipients had no documented history of prior SARS-CoV-2 infection, as confirmed by absence of SARS-CoV-2-specific antibodies on serial sampling since study enrolment. Sera was heat-inactivated at $56^{\circ} \mathrm{C}$ for $30 \mathrm{~min}$ to inactivate complement prior to micro-neutralization.

Determination of $50 \%$ tissue culture infective dose $\left(\right.$ TCID $\left._{50}\right)$. The viral $50 \%$ tissue culture infective dose $\left(\mathrm{TCID}_{50}\right)$ was determined for each variant virus. Briefly, a passage one aliquot of virus stock was serially diluted $\left(1 \times 10^{-2}-1 \times 10^{-7}\right)$ in virus inoculation media. Virus dilutions were used to inoculate VeroE6/TMPRSS2 cells at $60 \%$ confluence in Costar ${ }^{\circledR}$ 24-well clear tissue culture-treated multiple well plates (353072, Corning Inc.). in quad triplicate. Plates were sealed with AeraSeal ${ }^{\circledR}$ Film (Excel Scientific Inc.) to minimise evaporation, spillage, and well-to-well cross-contamination. Plates were inspected daily for CPE and $110 \mu \mathrm{L}$ sampled at inoculation, 48, 72 and $96 \mathrm{hrs}$. Infections were terminated at $96 \mathrm{hrs}$ based on visual inspection for CPE and used in conjunction with RT-qPCR results to determine each isolate's $\operatorname{TCID}_{50}$.

Micro-neutralization assay. VeroE6/TMPRSS2 cells were seeded with DMEM from stocks in Costar 96-well clear tissue culture-treated flat bottom plates at $40 \%$ confluence. Cells were incubated at $37^{\circ} \mathrm{C}$ with $5 \% \mathrm{CO}_{2}$ for $12 \mathrm{hrs}$ or until they reached $60 \%$ confluence. Virus stocks were diluted to $200 \mathrm{TCID}_{50}$ in inoculation media. Doubling dilutions from 1:10 to 1:320 of vaccine-naïve and post BNT162b2 vaccination sera were added in equal proportions with virus in a 96 well plate and incubated for $60 \mathrm{~min}$ at $37^{\circ} \mathrm{C}$ and $5 \% \mathrm{CO}_{2}$ to enable virus neutralization. After this incubation the media was removed from 
the cell monolayer and $100 \mu \mathrm{L}$ of fresh media was added. Each dilution of sera was performed in duplicate per virus variant; 12 wells of uninfected cells were used per plate as a negative control. Plates were sealed with AeraSeal ${ }^{\circledR}$ Film to minimise evaporation, spillage, and well-to-well crosscontamination. After 60 mins of viral neutralization a residual $110 \mu \mathrm{L}$ was sampled from the 8 naïve patient wells per virus for extraction and RT-qPCR. Plates were inspected daily for CPE with a final read recorded at $72 \mathrm{hrs}$ independently by three scientists. SARS-CoV-2 in-house RT-qPCR was used to quantify the viral load post-neutralization, with $110 \mu \mathrm{L}$ of each dilution removed at $72 \mathrm{hrs}$ to determine viral load. The $110 \mu \mathrm{L}$ of each dilution was added to $110 \mu \mathrm{L}$ of External Lysis buffer (06374913001) at a 1:1 ratio in a 96-well deep-well extraction plate, covered with a MagNA Pure Sealing Foil (06241603001), and left to rest in the biosafety class two cabinet for $10 \mathrm{mins}$, a time-period shown to inactivate SARS-CoV-2 by in-house verification of a published protocol. ${ }^{15}$ The RNA was then extracted with the Viral NA Small volume kit on the MagNA Pure 96 system (06 543588 001, Roche Diagnostics $\mathrm{GmbH}$ ). Median neutralization breakpoints (CPE) were calculated using the breakpoints for individual sera for all three SARS-CoV-2 variants.

Statistical Analysis. Mean neutralizing antibody titres (nAbT) were evaluated and statistical significance assessed using the $t$ test with a 2-tailed hypothesis. Results were considered statistically significant at $p<.05$. Graphs were generated using RStudio (version 3.6.1)

\section{Results}

\section{Levels of neutralizing antibody against different SARS-CoV-2 lineages}

Neutralizing antibody titres (nAbT) in sera examined four weeks after a third dose of BNT162b2 were higher than nAbT measured at 1, 3, and 6 months after two doses of BNT162b2 (Figure 1, Table S1). However, there was a 4-fold reduction in median nAbT against Omicron in contrast to the wild-type and 1.5-fold decrease of nAbT against Delta VOC following the third dose. Median nAbT after 1, 3, 6 months of BNT162b2 vaccination for all variants were documented with titers of $<10$ and $<20$ (Figure 1, Table S1). Trends in decreasing nAbT in sera collected 1, 3, and 6 month post-two doses of BNT162b2 were observed, comparable to decreasing trends in SARS-CoV-2-specific IgG levels determined by immunofluorescence antibody assays (IFA; Table S1, Figure S1). Increases in nAbT were noted four weeks after BNT162b2 boosters, with median titers of 240, 160 and 60 for wild-type, Delta and Omicron respectively. Although median responses were lower for Delta $(\mathrm{p}=0.28)$ and Omicron $(\mathrm{p}=0.55)$, this reduction did not reach statistical significance.

\section{Different in vitro infection kinetics between SARS-CoV-2 lineages}

Three lineages demonstrated comparable $50 \%$ tissue culture infective dose $\left(\mathrm{TCID}_{50}\right)$ and viral load at inoculation (Figure S2). Nevertheless, Omicron had a slower propagation rate with increases in viral 
load not detected in culture supernatant until 96 hours post-infection (Figure S2). In contrast, wild-type and Delta cultures showed 4-5 $\log _{10}$ increase in viral load 72 hours post-inoculation (Figure S3).

\section{Discussion}

Omicron has rapidly evolved to encode over 30 protein substitutions in the SARS-CoV-2 spike protein, raising grave concerns for COVID-19 vaccine effectiveness, vaccines which were designed using ancestral viruses. Although reductions in $\mathrm{nAbT}$ are noted when human sera from double-dose BNT162b2 vaccinated individuals were challenged with Omicron, sera after a third boosting dose neutralized SARS-CoV-2. However, the observation of delays in Omicron growth in vitro suggests that these mutational changes may negatively affect viral fitness. Further investigations are required to determine if the cell infection dynamics are due to reduced efficiency in viral entry or host cell egress, although others have noted that ACE2 is still required for efficient Omicron propagation in cell culture. ${ }^{16}$ Given the longer growth times, understanding viral kinetics of Omicron infections is important to guide infection control practices as the duration of infectivity, peak viral load and shedding may be different. Our findings also suggest that booster vaccines are likely to be required to minimize SARS-CoV-2 transmission, given that BNT162b2 is highly effective in preventing SARS-Cov-2 infection and mortality, particularly in those with waning immunity post primary vaccination. ${ }^{17}$ Despite the limited sample number, this study's strength is the serial sera samples from four participants collected pre-and post-booster. This report also lacked samples from persons previously infected with SARS-CoV-2, but previous studies have demonstrated comparable antibody levels to vaccine recipients after six months. ${ }^{14}$ Laboratory variables such as cell lines, SARS-CoV-2 variants, age groups and timing of sera collection (in relation to infection and/or vaccination) used, and test methods $\left(\mathrm{TCID}_{50}\right.$; incubation times; type of neutralization test [e.g. focus, plaque or microneutralization]) need to be considered when interpreting nAbT studies.

\section{Conclusion}

These initial findings suggest that Omicron can be neutralized by sera collected from BNT162b2 recipients, optimally after three vaccine doses. However, the ability to neutralize Omicron is reduced, suggesting that there may be high incidences of breakthrough infections or reinfections despite vaccine boosters. Further studies with larger and targeted cohorts (e.g. young, elderly or immunocompromised) are warranted, alongside investigations into Omicron's evasiveness to T-cell responses and primary and boosted vaccine effectiveness.

\section{References}


1. Network for Genomic Surveillance in South Africa (NGS-SA) SARS-CoV-2 Sequencing Update 1 December 2021. https://www.nicd.ac.za/wp-content/uploads/2021/12/Update-of-SAsequencing-data-from-GISAID-1-Dec-Final.pdf (2021).

2. Classification of Omicron (B.1.1.529): SARS-CoV-2 Variant of Concern. World Health Organization https://www.who.int/news/item/26-11-2021-classification-of-omicron(b.1.1.529)-sars-cov-2-variant-of-concern (2021).

3. Basile, K. et al. Cell-based culture of SARS-CoV-2 informs infectivity and safe de-isolation assessments during COVID-19. Clin. Infect. Dis. (2020) doi:10.1093/cid/ciaa1579.

4. Wang, Z. et al. mRNA vaccine-elicited antibodies to SARS-CoV-2 and circulating variants 2 3. bioRxiv 2021.01.15.426911 (2021) doi:10.1101/2021.01.15.426911.

5. Lam, C. et al. SARS-CoV-2 Genome Sequencing Methods Differ in Their Abilities To Detect Variants from Low-Viral-Load Samples. J. Clin. Microbiol. 59, (2021).

6. Freed, N. E., Vlková, M., Faisal, M. B. \& Silander, O. K. Rapid and inexpensive wholegenome sequencing of SARS-CoV-2 using 1200 bp tiled amplicons and Oxford Nanopore Rapid Barcoding. Biol. Methods Protoc. 5, (2021).

7. Rockett, R. J. et al. Revealing COVID-19 transmission in Australia by SARS-CoV-2 genome sequencing and agent-based modeling. Nat. Med. 26, 1398-1404 (2020).

8. Bolger, A. M., Lohse, M. \& Usadel, B. Trimmomatic: A flexible trimmer for Illumina sequence data. Bioinformatics 30, 2114-2120 (2014).

9. Li, H. \& Durbin, R. Fast and accurate short read alignment with Burrows-Wheeler transform. Bioinformatics 25, 1754-1760 (2009).

10. Grubaugh, N. D. et al. An amplicon-based sequencing framework for accurately measuring intrahost virus diversity using PrimalSeq and iVar. Genome Biol. 20, 8 (2019).

11. Turakhia, Y. et al. Stability of SARS-CoV-2 phylogenies. PLOS Genet. 16, e1009175 (2020).

12. O'Toole, Á. et al. Assignment of epidemiological lineages in an emerging pandemic using the pangolin tool. Virus Evol. 7, (2021).

13. Rambaut, A. et al. A dynamic nomenclature proposal for SARS-CoV-2 lineages to assist genomic epidemiology. Nat. Microbiol. 5, 1403-1407 (2020).

14. Hueston, L. et al. The Antibody Response to SARS-CoV-2 Infection. Open Forum Infect. Dis. 7, (2020).

15. SARS-CoV-2 inactivation testing: interim report. https://assets.publishing.service.gov.uk/government/uploads/system/uploads/attachment_data/f ile/898618/HCM-CoV2-014-v3_40__GHCl_TCF.pdf (2020).

16. Cele, S. et al. SARS-CoV-2 Omicron has extensive but incomplete escape of Pfizer BNT162b2 elicited neutralization and requires ACE2 for infection.

17. Arbel, R. et al. BNT162b2 Vaccine Booster and Mortality Due to Covid-19. N. Engl. J. Med. 1-8 (2021) doi:10.1056/NEJMoa2115624.

\section{Acknowledgements:}

The authors acknowledge the technical assistance provided by the Sydney Informatics Hub, a Core Research Facility of the University of Sydney, the COVID Heroes (A serosurvey of healthcare workers caring for, or handling specimens from, individuals exposed to, or diagnosed with, SARS-CoV-2 infection in Australia) study participants from where the sera were sourced, the laboratories who referred samples for testing that were included in this analysis.

\section{Ethics:}

Ethical and governance approval for the study was granted by the Western Sydney Local Health District Human Research Ethics Committee (2020/ETH02426) and (2020/ETH00786).

\section{Disclaimer:}


The authors have no conflicts of interest to declare. All authors had full access to study data and final responsibility and approval of the version submitted for publication.

\section{Financial support:}

This study was supported by the Prevention Research Support Program funded by the NSW Ministry of Health, NSW Health COVID-19 priority funding, National Health and Medical Research Council, Australia (APPRISE 1116530). Dr Basile is supported by a Jerry Koutts PhD Scholarship from the ICPMR Trust Fund.

Figure 1. Increases in neutralizing antibody titers four weeks after the third dose of BNT162b2
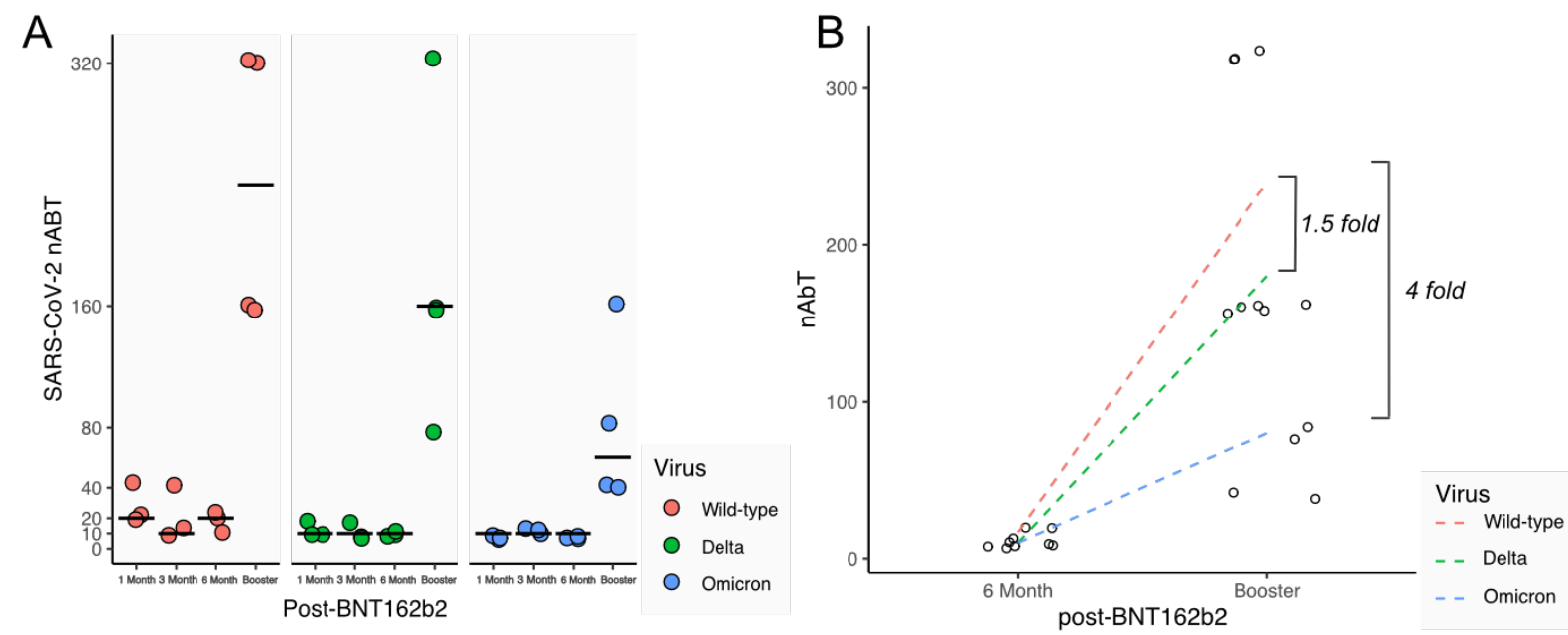

Figure 1A. Illustrates the neutralizing antibody titer of SARS-CoV-2 variants Delta (green) and Omicron (blue) compared to wild-type (red, SARS-CoV-2 lineage A.2.2) black lines indicate the median titre at each timepoint. B) The sera from four individuals collected six months after two doses of BNT162b2 and four weeks after a third boosting dose demonstrate an increase in neutralizing antibody titers against all three variants. However, a 1.5 and 4-fold reduction in neutralizing antibody titer is observed for the Delta and Omicroncompared to wild-type virus. Dashed lines depict the linear regression between individual titers pre- and post-booster for each virus. 